\title{
Electromyostimulation influences the mechanical properties and microarchitectures of bones beyond the stimulation site
}

\author{
Ishak S. LIMBONG **,***, Tomoki YAMAMURA ** and Shigeo M. TANAKA* \\ * Institute of Science and Engineering, Faculty of Frontier Engineering, Kanazawa University, \\ Kakuma-machi, Kanazawa, Ishikawa 920-1192, Japan. \\ E-mail: shigeo@se.kanazawa-u.ac.jp \\ ** Graduate School of Natural Science and Technology, Division of Mechanical Science and Engineering, Kanazawa University, \\ Kakuma-machi, Kanazawa, Ishikawa 920-1192, Japan. \\ *** Mechanical Engineering Department, Faculty of Science and Engineering, Nusa Cendana University, \\ Kupang, Nusa Tenggara Timur 85228, Indonesia.
}

Received: 10 January 2019; Revised: 24 April 2019; Accepted: 21 May 2019

\begin{abstract}
Electromyostimulation is a nonpharmacological prevention method for osteoporosis that is safe and feasible for the elderly and people with physical disabilities. Our previous study demonstrated that random pulse train (RdPT) electromyostimulation of rat quadriceps induces an increase in the mechanical properties of the contralateral unstimulated femoral neck. However, the efficacy of this stimulation on other untested bones is still unclear. The objective of this research is to investigate the response of previously unstimulated bones to single-site electromyostimulation. The left quadriceps of rats were stimulated electrically by periodic pulse train (PrPT) or RdPT with $2 \mathrm{~mA}$-magnitude pulses at $552 \mu$ s and a 50\% duty ratio. The stimulation effect was examined on the diaphysis of long bones and lumbar vertebrae (L2-L5) by quasi-static mechanical tests and microcomputed tomography analysis. RdPT increased the strain energy at the stimulated left femur but did not change the properties of the other long bones. For the lumber vertebrae, on the other hand, both stimulations showed similar results. The stiffness of lumbar vertebra increased in L2, and the stiffness and the maximum load decreased in L4. Additionally, the BMC (bone mineral content), BV (bone volume), and TV (tissue volume) were reduced in L2, but not changed in L4. The other vertebrae were not affected by the stimulations. In conclusion, RdPT influences not only the stimulated femur, but also the lumbar vertebrae site-dependently as well as PrPT. These findings suggest the whole-body scale effect of electromyostimulation, however, which is not positive in all the bones, requiring further investigations for its clinical applications.
\end{abstract}

Keywords: Electromyostimulation, Random stimulation, Osteoporosis, Mechanical properties, Bone microarchitecture

\section{Introduction}

Osteoporosis is a progressive disease characterized by decreasing bone mineral density (BMD) or bone microarchitecture deterioration with aging, which eventually results in bone fracture. Osteoporotic fracture in elderly people reduces their quality of life and life expectancy. In most cases, osteoporosis is not properly diagnosed until a bone is broken because of the lack of other symptoms. There are many studies investigating the treatment and prevention of osteoporosis, including pharmacological approaches. Drugs are used as a general treatment but can cause side effects (Gourlay et al., 2003). In addition to pharmaceutical treatments, physical activities such as walking, running, and jumping are often recommended clinically to maintain bone density and prevent osteoporosis, although the osteogenic effect of mechanical loading differs by age (Plochocki, 2009) and activity type (Shibata et al., 2003). However, these mechanical loading activities can be difficult for elderly people who have poor locomotive ability and 
are potentially not feasible in some special circumstances, e.g., for bedridden patients or astronauts in a low gravity environment. Therefore, alternative methods are required to achieve the beneficial effect of physiotherapy in difficult conditions and prevent osteoporosis.

Electromyostimulation has been previously studied as an alternative to mechanical stimulation in the prevention of osteoporosis (Lam and Qin, 2008). Electromyostimulation has been reported to induce muscle contraction and mechanical force in the bone via tendons (Tanaka and Kondo, 2009), thus resulting in increased bone formation (Tanaka, 2014), bone loss suppression under unloading conditions (Lam and Qin, 2008), or decreased muscle mass loss in denervation conditions (Tamaki et al., 2017). Although the interactions between bone and muscle are still unclear, previous studies demonstrated that muscle contraction influences not only mechanical conditions but also blood circulation (Caulkins et al., 2009) and endocrine activity (Elkasrawy and Hamrick, 2010) in bones and muscles. Our previous study demonstrated that electromyostimulation-induced contraction forces are influenced by the frequency and resting time of electrical stimulation (Tanaka and Kondo, 2009). This influence is related to muscle fatigue during stimulation (Matsunaga et al., 1999). A critical factor in eliciting significant osteogenesis is the stimulation pattern of bones by muscle contraction; however, this topic is controversial because it likely has strong nonlinearity between the stimulation amount and the effectiveness (Tanaka et al., 2002; Turner, 1998).

Random electrical pulse train (RdPT) is a new pattern of electromyostimulation (Takimoto and Tanaka, 2010) that increases the mechanical properties not only of the stimulated side of the femora neck but also of the unstimulated contralateral femora neck after quadricep stimulation in rats (Tanaka et al., 2017). However, periodic electrical pulse train (PrPT) at a fixed repetition frequency of $20 \mathrm{~Hz}$ did not show any physiological changes at these sites. It is possible that RdPT is effective in other bones beyond the stimulation site on a whole-body scale. The objective of this study is to identify if these electromyostimulations are capable of enhancing the mechanical properties of bones not only in the femoral stimulation site but also beyond the stimulated site, e.g., in the tibia, humerus, ulna-radius, or lumbar vertebra, in a rat model.

\section{Materials and Methods}

\subsection{Animals}

A total of 30 female Sprague-Dawley rats (7 weeks old, $145.77 \pm 7.08 \mathrm{~g}$ weight) were purchased and housed under standard laboratory condition and provided with free access to food and water. They were randomly divided into three groups (Control, PrPT, and RdPT) with 10 rats in each, where 5 rats were used for investigation of the stimulation effects on long bones, and the other 5 rats for vertebrae, based on our previous study (Tanaka et al., 2017). In Control group, one rat was lost by death due to unknown cause. The animals were acclimated to the laboratory environment (12 $\mathrm{h}$ day-night cycle and $24^{\circ} \mathrm{C}$ temperature) a week before stimulation. The animals were divided into three groups: the age-matched sham (Control) group, PrPT electromyostimulation group, and RdPT electromyostimulation group. Electrode insertion was also performed on the sham control rats over the stimulation time but without stimulation to confirm that there is no inflammation or mechanical injury due to electrode insertion, which could affect osteogenesis. All the experimental procedures were approved by the Committee on Animal Experimentation of Kanazawa University (Approval No. AP-173865) and were performed according to the guidelines provided by the Institute for Experimental Animals of Kanazawa University.

\subsection{Electromyostimulation and bone harvesting}

The electromyostimulation of rat quadriceps was performed as described previously (Tanaka, 2014; Tanaka et al., 2017; Tanaka and Kondo, 2009). Prior to stimulation, the rats were anesthetized with an intraperitoneal injection of pentobarbital sodium. PrPT or RdPT electrical signals composed of $2 \mathrm{~mA}$-magnitude pulses at $552 \mu$ s and a $50 \%$ duty ratio were generated by a personal computer with Windows OS. The electrical signals were then sent to stainless steel needle electrodes inserted into the left quadriceps of the rats for $30 \mathrm{~min}$ a day for three consecutive days. Fifteen days after the last stimulation day, the rats were sacrificed, and the femurs, tibiae, humeri, ulnas-radii, and lumbar vertebrae L2, L3, L4, and L5 were harvested. These bones were cleaned from their soft tissue, stored in saline water, and immediately tested mechanically by using a universal mechanical testing machine (AG-Xplus, Shimadzu, Kyoto, Japan) with a 20kN load cell (Kit number 346-5670431, Shimadzu, Kyoto, Japan). 


\subsection{Mechanical test}

The mechanical properties of the femurs, tibiae, humeri, and ulnas-radii were evaluated by the four-point bending test. The bones were placed on the $16 \mathrm{~mm}$-span bottom jig and pressed with an $8 \mathrm{~mm}$-span jig with a displacement speed of $1 \mathrm{~mm} / \mathrm{min}$ until broken in the posterior-anterior direction for the femurs and the lateral-medial direction for the tibiae, humeri, and ulnas-radii. Maximum load, strain energy, and stiffness were obtained from load-displacement curves. Alternatively, the lumbar vertebrae were tested by a compression test wherein the lumbar vertebrae were placed on the bottom of a compression plate in the direction of the bone axis. Their extended arches were removed, thus making their superior and inferior surfaces parallel. The compression tests were performed at a speed of $1 \mathrm{~mm} / \mathrm{min}$ until a $5 \mathrm{~mm}$ displacement was observed. The maximum load, strain energy, and stiffness at a displacement less than 4 $\mathrm{mm}$, which is approximately half of the vertebrae height, were obtained from the load-displacement curves. In both type of tests, stiffnesses were calculated with a linier regression's slope of the initial linear region (Fig. 1).

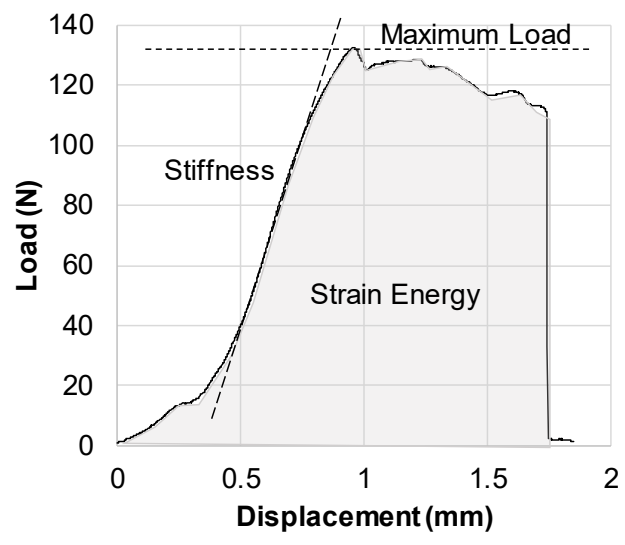

(a) long bone (femur)

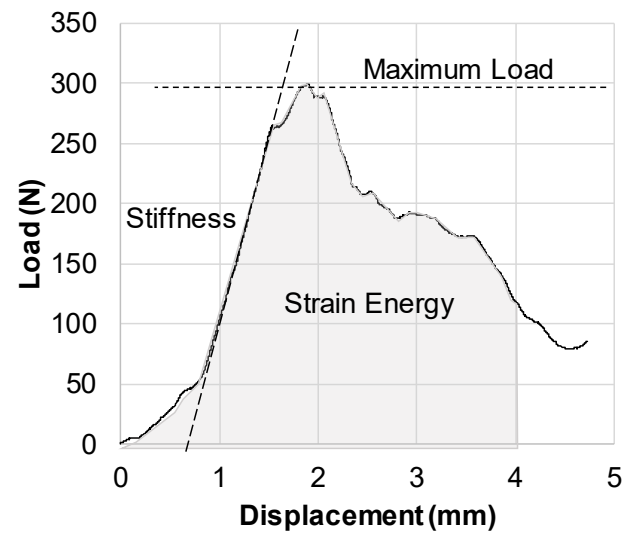

(b) vertebra (L2)

Fig. 1 Typical load-displacement curves of bending test on a long bone (a), and compression test on a vertebra (b). Stiffness was calculated with a linier regression's slope of the initial linear region. Strain energy was evaluated at a breaking point in the bending test and at 4-mm displacement in the compression test.

\subsection{Microcomputed tomography}

The lumbar vertebrae were scanned using a microcomputed tomography $(\mu \mathrm{CT}$ ) system (InspeXio SMX-90CT Plus with HPC InspeXio high-performance computing system, Shimazu, Kyoto, Japan) before they were tested mechanically. Every scanning day, the vertebrae images were calibrated using the Phantom Ratoc system with a five-cylinder calibration phantom, which has a set of graduated mineral concentrations of 100, 300, 400, 200, and 500 mg/cm3 (1508-113_No06_U5D1 mmH, Ratoc System Engineering, Tokyo, Japan). The scanning process was set to a resolution of $0.040 \mathrm{~mm} /$ pix and machine conditions of $90 \mathrm{kV}$ and $110 \mu \mathrm{A}$. From the $\mu \mathrm{CT}$ images, BMD, volumetric BMD (vBMD), bone mineral content (BMC), bone volume (BV), and tissue volume (TV) were analyzed using a 3D bone morphometric analysis software (TRI/3D-BON, Ratoc System Engineering, Tokyo, Japan).

\subsection{Data analysis}

To assess the statistical significance of the effectiveness of electromyostimulation treatment among the groups, the results were analyzed with Kaleidagraph Software (Version 3.6; Synergy Software, PA, USA). ANOVA with Dunnett's post-hoc tests were performed to compare the three experimental groups. Paired t-tests were conducted to compare the left and right bones. A p value of 0.05 or lower indicated statistical significance.

\section{Results}

\subsection{Body weight}

The body weight of the animals did not show any significant differences among the different treatment groups on the first day of stimulation, with average weights of $171.75 \pm 10.72,172.70 \pm 12.22$, and $171.05 \pm 6.06 \mathrm{~g}$ for the control, PrPT, and RdPT groups, respectively. Furthermore, the body weights were not significantly different on the day of sacrifice: $212.13 \pm 14.73,210.76 \pm 10.42$, and $208.09 \pm 9.09 \mathrm{~g}$ for the control, PrPT, and RdPT groups, respectively. 
These results show the equality of conditions of all rats even in different treatment groups and that electromyostimulation did not affect their body weights.

\subsection{Mechanical properties of the diaphysis of the long bones}

Four-point bending testing on the diaphysis of the femurs showed that the strain energy of RdPT-stimulated left femurs has a significantly larger increase than that of unstimulated contralateral right femurs by $46.32 \%(p<0.05)$ (Fig. 2). Other mechanical properties such as the maximum load and stiffness of the femur did not show significant differences between the left and right sides. Furthermore, electromyostimulation to the left quadriceps did not significantly influence the mechanical properties of the diaphysis of the other long bones, namely, the tibiae, humeri, and ulnas-radii (Table 1). However, in the case of an incomplete fracture of the ulna-radius, the strain energy could not be analyzed (data unavailable).

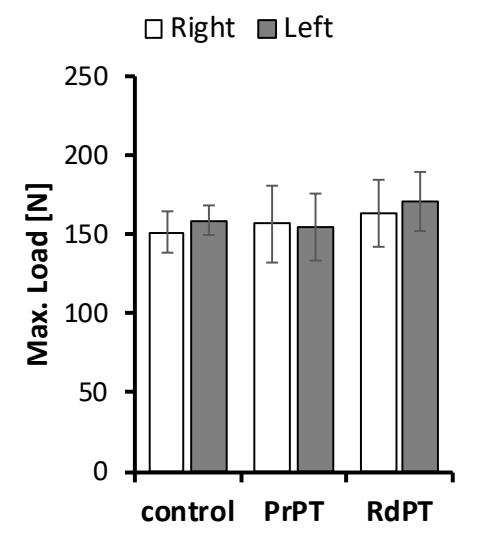

(a) maximum load of femur

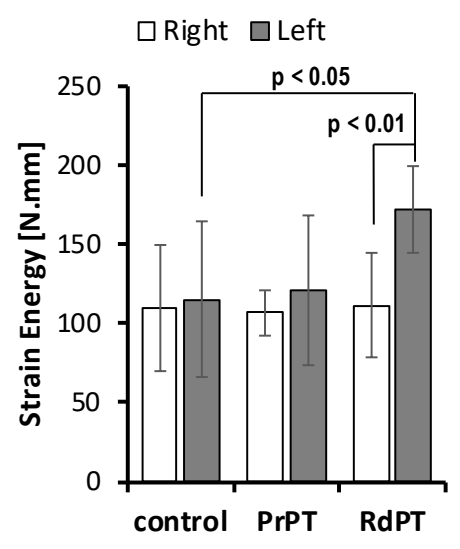

(b) strain energy of femur

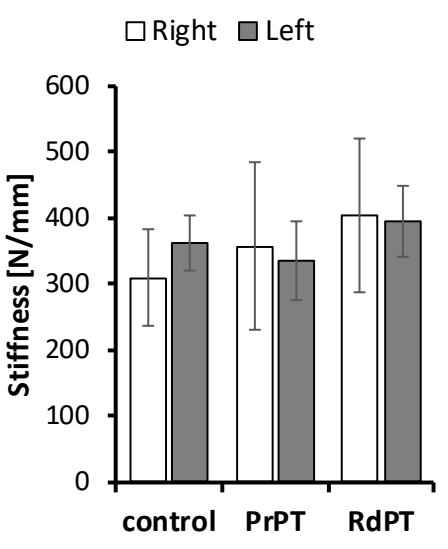

(c) stiffness of femur

Fig. 2 Comparison of the mechanical properties of the diaphysis of the femur: (a) maximum load, (b) strain energy, and (c) stiffness. The strain energy of the RdPT-stimulated left femurs was significantly increased by the stimulation compared with those of the control left femurs or RdPT-unstimulated right femur. The value and error bar show the average and standard deviation of five samples.

Table 1 Comparison of the mechanical properties of the femur, tibia, humerus, and ulna-radius diaphysis after the electromyostimulation of the left quadriceps. The stimulation did not induce significant changes in the bone mechanical properties, except the strain energy of the stimulated left femur diaphysis. The value and error bar show the average and standard deviation of five samples. ${ }^{*} \mathrm{p}<0.05$ vs. control; ${ }^{++} \mathrm{p}<0.01$ vs. contralateral; N/A: not available because no fracture point was observed.

\begin{tabular}{|c|c|c|c|c|c|c|c|}
\hline & & \multicolumn{2}{|c|}{ Control $(n=5)$} & \multicolumn{2}{|c|}{$\operatorname{PrPT}(n=5)$} & \multicolumn{2}{|c|}{$\operatorname{RdPT}(n=5)$} \\
\hline & & Right & Left & Right & Left & Right & Left \\
\hline \multirow[t]{3}{*}{ Femur } & Max. Load (N) & $151.3 \pm 12.9$ & $158.7 \pm 9.4$ & $156.6 \pm 24.0$ & $154.7 \pm 21.6$ & $163.2 \pm 21.6$ & $171.3 \pm 18.7$ \\
\hline & Strain Energy $(\mathrm{N} \cdot \mathrm{mm})$ & $109.5 \pm 40.3$ & $115.2 \pm 49.3$ & $106.7 \pm 13.8$ & $121.1 \pm 47.0$ & $111.6 \pm 33.0$ & $172 \pm 27.5^{\star^{++}}$ \\
\hline & Stiffness (N/mm) & $308.8 \pm 72.3$ & $361.2 \pm 41.4$ & $357 \pm 125.7$ & $335.6 \pm 60.0$ & $403.4 \pm 117.7$ & $394.4 \pm 52.8$ \\
\hline \multirow[t]{3}{*}{ Tibia } & Max. Load (N) & $110.2 \pm 28.0$ & $113.4 \pm 17.1$ & $126.4 \pm 33.8$ & $122.1 \pm 16.4$ & $126 \pm 17.9$ & $116.4 \pm 17.2$ \\
\hline & Strain Energy $(\mathrm{N} \cdot \mathrm{mm})$ & $120.1 \pm 56.2$ & $95.39 \pm 48.33$ & $170 \pm 75.5$ & $125.2 \pm 40.7$ & $111.4 \pm 31.6$ & $108.9 \pm 34.4$ \\
\hline & Stiffness (N/mm) & $194.4 \pm 57.9$ & $186.5 \pm 37.7$ & $204.4 \pm 65.7$ & $203.5 \pm 42.2$ & $193.4 \pm 24.0$ & $148.8 \pm 49.8$ \\
\hline \multirow[t]{3}{*}{ Humerus } & Max. Load (N) & $75.68 \pm 12.03$ & $69.52 \pm 20.80$ & $73.48 \pm 12.90$ & $84.73 \pm 15.72$ & $77.65 \pm 15.58$ & $81.49 \pm 13.35$ \\
\hline & Strain Energy (N.mm) & $42.75 \pm 26.20$ & $53.17 \pm 12.56$ & $47.28 \pm 20.57$ & $44.73 \pm 23.04$ & $48.5 \pm 21.44$ & $52.74 \pm 21.76$ \\
\hline & Stiffness (N/mm) & $165.5 \pm 46.9$ & $118.3 \pm 57.7$ & $144.1 \pm 63.4$ & $185.7 \pm 38.6$ & $163.1 \pm 41.9$ & $157.3 \pm 69.4$ \\
\hline \multirow[t]{3}{*}{ Ulna-Radiu } & Max. Load (N) & $57.12 \pm 3.44$ & $51.31 \pm 7.44$ & $58.53 \pm 9.14$ & $50.23 \pm 7.21$ & $56.97 \pm 1.74$ & $55.73 \pm 6.94$ \\
\hline & Strain Energy (N.mm) & $\mathrm{N} / \mathrm{A}$ & $\mathrm{N} / \mathrm{A}$ & $\mathrm{N} / \mathrm{A}$ & $\mathrm{N} / \mathrm{A}$ & $\mathrm{N} / \mathrm{A}$ & $\mathrm{N} / \mathrm{A}$ \\
\hline & Stiffness (N/mm) & $61.78 \pm 7.87$ & $57.47 \pm 12.68$ & $47.99 \pm 15.44$ & $55.58 \pm 6.37$ & $54.07 \pm 13.02$ & $59.94 \pm 17.35$ \\
\hline
\end{tabular}




\subsection{Mechanical properties of the lumbar vertebrae}

Compression testing on the L2 demonstrated a significant increase in bone rigidity in the RdPT group by $53.90 \%$ ( $\mathrm{p}<0.05$ ), also an increase in the PrPT group by $17.57 \%$ compared with the control (Fig. 3). However, RdPT stimulation significantly decreased the stiffness of the L4 by $39.22 \%$ ( $p<0.05$ ), and the maximum load by $8.39 \%$ compared with the nonstimulated control (Table 2). PrPT stimulation also decreased the stiffness of L4 by $11.11 \%$ and significantly reduced the maximum load by $13.32 \%(\mathrm{p}<0.05)$. Furthermore, no significant changes in mechanical properties were observed in the L3 or L5 after the stimulation.

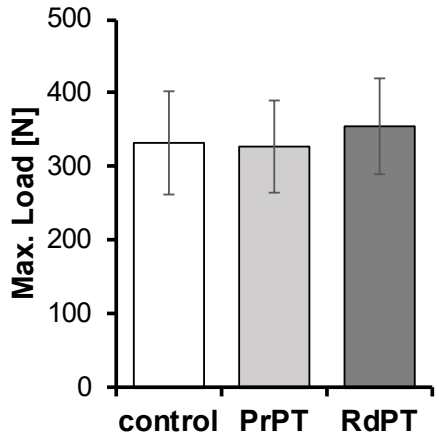

(a) maximum load of $\mathrm{L} 2$

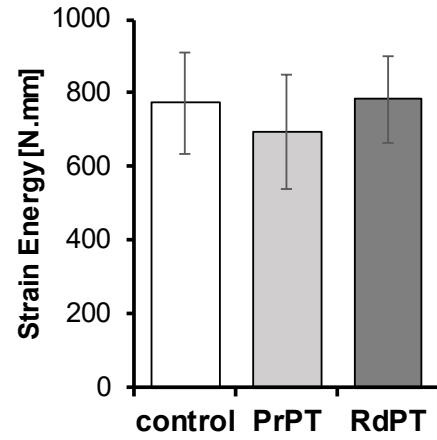

(b) strain energy of $L 2$

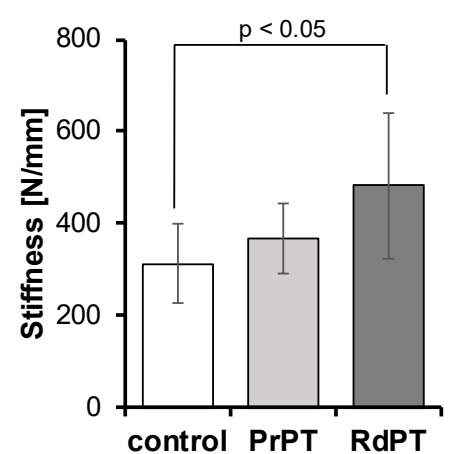

(c) stiffness of L2

Fig. 3 Comparison of the mechanical properties of the L2 vertebrae: (a) maximum load, (b) strain energy, and (c) stiffness. The stiffness of the RdPT group was increased significantly by electromyostimulation to the left quadriceps. The value and error bar show the average and standard deviation of four samples for Control, five samples for PrPT and PdPT.

Table 2 Comparison of the mechanical properties of the L2, L3, L4, and L5 vertebrae. The mechanical properties did not change as a result of electromyostimulation to the left quadriceps, except the stiffness of L2 and L4 in the RdPT group and the maximum load of L4 in the PrPT group. The average and standard deviation of four or five samples were indicated for Control or for PrPT and PdPT, respectively. ${ }^{*} \mathrm{p}<0.05$ vs. control.

\begin{tabular}{|c|c|c|c|c|}
\hline & & Control $(n=4)$ & $\operatorname{PrPT}(n=5)$ & $\operatorname{RdPT}(n=5)$ \\
\hline \multirow[t]{3}{*}{ L2 } & Max. Load (N) & $331.8 \pm 70.6$ & $327.1 \pm 62.6$ & $335.6 \pm 65.2$ \\
\hline & Strain Energy (N.mm) & $772.3 \pm 136.0$ & $693.9 \pm 156.9$ & $782.6 \pm 118.4$ \\
\hline & Stiffness (N/mm) & $313.0 \pm 86.2$ & $368.0 \pm 77.3$ & $481.7 \pm 159.2^{*}$ \\
\hline \multirow[t]{3}{*}{ L3 } & Max. Load (N) & $380.9 \pm 17.3$ & $334.9 \pm 66.7$ & $334.6 \pm 70.4$ \\
\hline & Strain Energy (N.mm) & $890.0 \pm 89.0$ & $758.2 \pm 164.7$ & $743.2 \pm 111.4$ \\
\hline & Stiffness (N/mm) & $403.5 \pm 75.8$ & $351.2 \pm 136.8$ & $333.6 \pm 137.9$ \\
\hline \multirow[t]{3}{*}{ L4 } & Max. Load (N) & $404.0 \pm 20.8$ & $350.2 \pm 44.9^{*}$ & $370.1 \pm 57.5$ \\
\hline & Strain Energy (N.mm) & $799.1 \pm 41.4$ & $770.4 \pm 100.2$ & $808.9 \pm 146.9$ \\
\hline & Stiffness (N/mm) & $476.3 \pm 120.4$ & $423.4 \pm 109.9$ & $289.5 \pm 112.7^{*}$ \\
\hline \multirow[t]{3}{*}{ L5 } & Max. Load (N) & $349.0 \pm 31.1$ & $351.9 \pm 23.6$ & $361.2 \pm 19.8$ \\
\hline & Strain Energy $(\mathbf{N} \cdot \mathbf{m m})$ & $778.3 \pm 93.7$ & $825.2 \pm 30.5$ & $791.8 \pm 111.9$ \\
\hline & Stiffness (N/mm) & $319.1 \pm 28.7$ & $393.8 \pm 121.6$ & $327.5 \pm 72.8$ \\
\hline
\end{tabular}

\subsection{Bone microarchitecture of the lumbar vertebrae}

Figure 4 shows the representative microstructure of the L2, the findings suggested that no remarkable differences existed among the groups. Fig. 5 presents a comparison of the microstructure parameters of the L2 among the groups as a representative of the vertebrae. There was no significant difference between the BMD and vBMD in the L2 in the groups. Significant differences were observed in the other microstructural parameters of the L2. RdPT and PrPT 
reduced the BMC of the L2 by $6.90 \%(\mathrm{p}<0.05)$ and $7.30 \%(\mathrm{p}<0.05)$, respectively, compared with the control. These stimulation-induced decreases were also observed in the BV and TV of the L2. However, there were no significant differences in the other lumbar vertebrae when observing the microstructural parameters among the groups (Table 3).
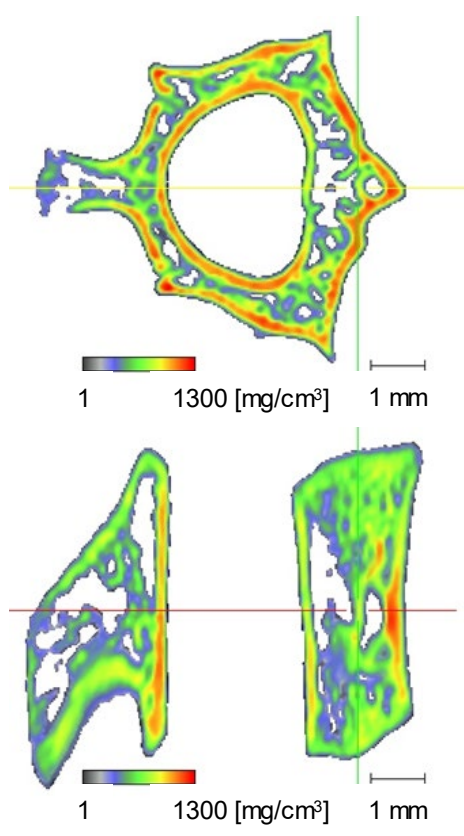

(a) control (L2)
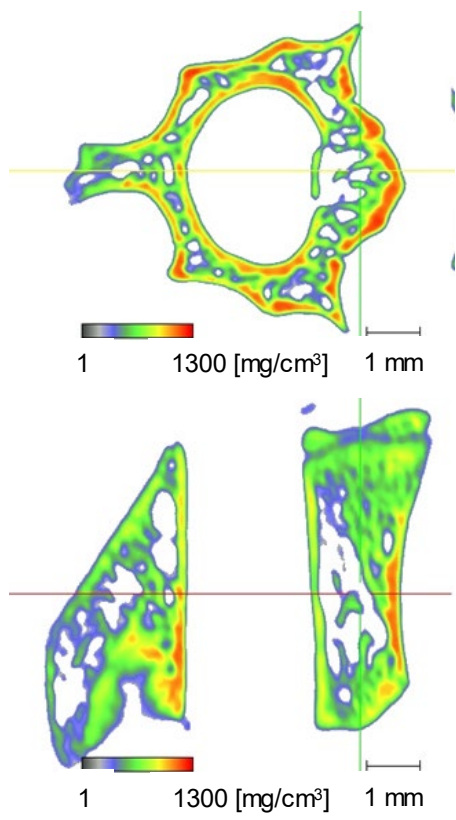

(b) PrPT (L2)
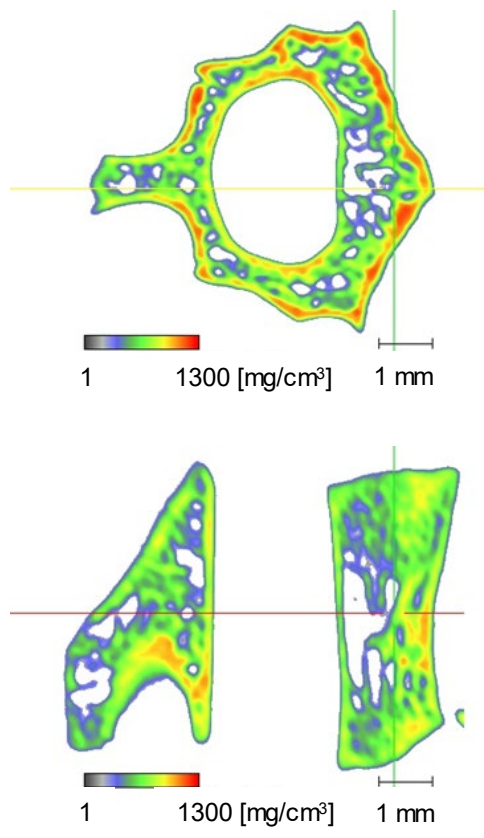

(c) RdPT (L2)

Fig. 4 Representative bone structure images of the L2 vertebrae on the transverse (upper) and sagittal (bottom) planes in the (a) control, (b) PrPT, and (c) RdPT groups. There are no obvious differences among the groups.

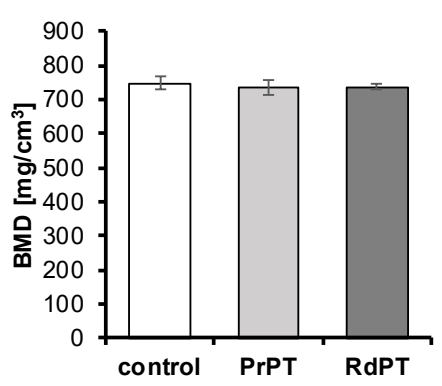

(a) BMD of L2

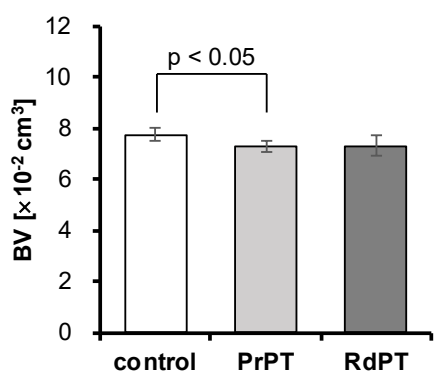

(d) BV of L2

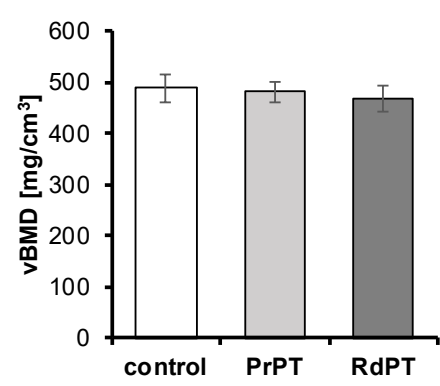

(b) VBMD of L2

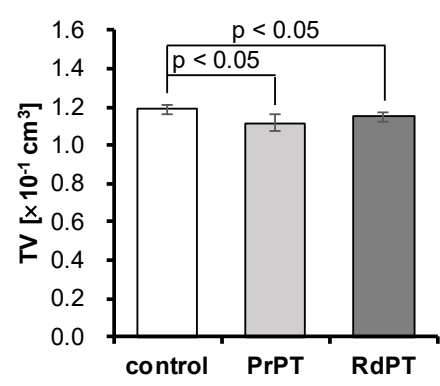

(e) TV of L2

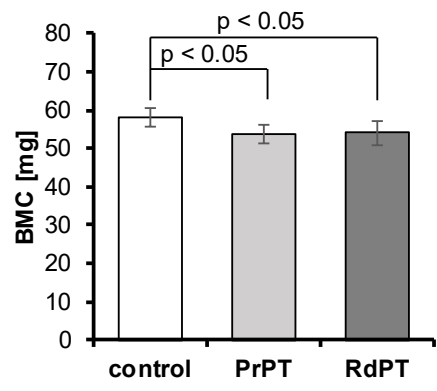

(c) BMC of L2

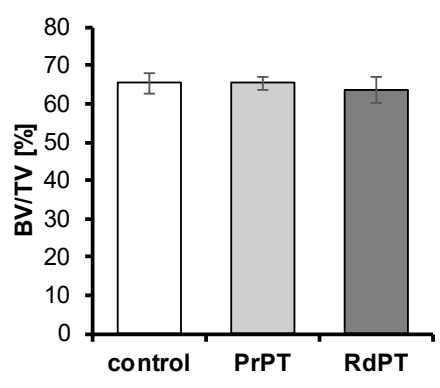

(f) BV/TV of L2

Fig. 5. Comparison of the microarchitectural parameters of the L2 vertebrae between the treatment groups: (a) BMD, (b) vBMD, (c) BMC, (d) BV, (e) TV, and (f) BV/TV fraction. The BMD, vBMD, and BV/TV were not influenced by electromyostimulation at the quadriceps. However, the BMC, BV, and TV decreased as a result of stimulation. The value and error bar show the average and standard deviation of four samples for Control, five samples for PrPT and PdPT. 
Table 3 Comparison of the microarchitecture of L2, L3, L4, and L5. The stimulation influenced only the microarchitecture of L2 but not those of L3, L4, and L5. The average and standard deviation of four or five samples were indicated for Control or for PrPT and PdPT, respectively. $* \mathrm{p}<0.05$ vs. control.

\begin{tabular}{|c|c|c|c|c|}
\hline & & Control $(n=4)$ & $\operatorname{PrPT}(n=5)$ & $\operatorname{RdPT}(n=5)$ \\
\hline \multirow[t]{6}{*}{ L2 } & $\operatorname{BMD}\left(\times 10^{2} \mathrm{mg} / \mathrm{cm}^{3}\right)$ & $7.48 \pm 0.15$ & $7.36 \pm 0.19$ & $7.38 \pm 0.07$ \\
\hline & $\operatorname{vBMD}\left(\times 10^{2} \mathrm{mg} / \mathrm{cm}^{3}\right)$ & $4.89 \pm 0.23$ & $4.81 \pm 0.18$ & $4.70 \pm 0.23$ \\
\hline & BMC (× $10 \mathrm{mg})$ & $5.81 \pm 0.19$ & $5.38 \pm 0.21^{*}$ & $5.41 \pm 0.28^{*}$ \\
\hline & $\operatorname{BV}\left(\times 10^{-2} \mathrm{~cm}^{3}\right)$ & $7.76 \pm 0.24$ & $7.31 \pm 0.19^{*}$ & $7.33 \pm 0.38$ \\
\hline & $\operatorname{TV}\left(\times 10^{-1} \mathrm{~cm}^{3}\right)$ & $1.19 \pm 0.02$ & $1.12 \pm 0.04^{*}$ & $1.15 \pm 0.02^{*}$ \\
\hline & BV/TV (\%) & $65.4 \pm 2.4$ & $65.4 \pm 1.4$ & $63.7 \pm 2.9$ \\
\hline \multirow[t]{6}{*}{ L3 } & BMD $\left(\times 10^{2} \mathrm{mg} / \mathrm{cm}^{3}\right)$ & $7.43 \pm 0.13$ & $7.33 \pm 0.21$ & $7.38 \pm 0.08$ \\
\hline & $\operatorname{vBMD}\left(\times 10^{2} \mathrm{mg} / \mathrm{cm}^{3}\right)$ & $5.01 \pm 0.25$ & $4.91 \pm 0.19$ & $4.94 \pm 0.26$ \\
\hline & BMC (× $10 \mathrm{mg})$ & $5.93 \pm 0.18$ & $5.81 \pm 0.21$ & $5.91 \pm 0.51$ \\
\hline & $\operatorname{BV}\left(\times 10^{-2} \mathrm{~cm}^{3}\right)$ & $7.97 \pm 0.14$ & $7.93 \pm 0.39$ & $8.01 \pm 0.68$ \\
\hline & $\operatorname{TV}\left(\times 10^{-1} \mathrm{~cm}^{3}\right)$ & $1.18 \pm 0.03$ & $1.18 \pm 0.07$ & $1.20 \pm 0.07$ \\
\hline & BV/TV (\%) & $67.3 \pm 2.3$ & $67.0 \pm 1.6$ & $66.9 \pm 2.9$ \\
\hline \multirow[t]{6}{*}{ L4 } & $\operatorname{BMD}\left(\times 10^{2} \mathrm{mg} / \mathrm{cm}^{3}\right)$ & $7.45 \pm 0.18$ & $7.41 \pm 0.14$ & $7.39 \pm 0.10$ \\
\hline & $\operatorname{vBMD}\left(\times 10^{2} \mathrm{mg} / \mathrm{cm}^{3}\right)$ & $5.17 \pm 0.30$ & $5.12 \pm 0.10$ & $5.08 \pm 0.25$ \\
\hline & BMC (× $10 \mathrm{mg})$ & $6.26 \pm 0.23$ & $6.11 \pm 0.25$ & $6.27 \pm 0.46$ \\
\hline & $\operatorname{BV}\left(\times 10^{-2} \mathrm{~cm}^{3}\right)$ & $8.41 \pm 0.25$ & $8.25 \pm 0.29$ & $8.49 \pm 0.59$ \\
\hline & $\operatorname{TV}\left(\times 10^{-1} \mathrm{~cm}^{3}\right)$ & $1.21 \pm 0.04$ & $1.19 \pm 0.04$ & $1.23 \pm 0.06$ \\
\hline & BV/TV (\%) & $69.3 \pm 2.6$ & $69.1 \pm 0.7$ & $68.6 \pm 2.5$ \\
\hline \multirow[t]{6}{*}{ L5 } & $\operatorname{BMD}\left(\times 10^{2} \mathrm{mg} / \mathrm{cm}^{3}\right)$ & $7.20 \pm 0.17$ & $7.29 \pm 0.18$ & $7.27 \pm 0.05$ \\
\hline & $\operatorname{vBMD}\left(\times 10^{2} \mathrm{mg} / \mathrm{cm}^{3}\right)$ & $5.19 \pm 0.27$ & $5.26 \pm 0.16$ & $5.25 \pm 0.21$ \\
\hline & BMC (× $10 \mathrm{mg})$ & $6.48 \pm 0.35$ & $6.28 \pm 0.13$ & $6.49 \pm 0.47$ \\
\hline & BV $\left(\times 10^{-2} \mathrm{~cm}^{3}\right)$ & $9.00 \pm 0.45$ & $8.63 \pm 0.29$ & $8.92 \pm 0.61$ \\
\hline & $\operatorname{TV}\left(\times 10^{-1} \mathrm{~cm}^{3}\right)$ & $1.25 \pm 0.06$ & $1.20 \pm 0.05$ & $1.23 \pm 0.05$ \\
\hline & BV/TV (\%) & $72.0 \pm 2.2$ & $72.1 \pm 1.3$ & $72.1 \pm 2.4$ \\
\hline
\end{tabular}

\section{Discussion}

Electromyostimulation has been previously reported to induce osteogenesis or increase the mechanical properties of the stimulated bone (Lam and Qin, 2008; Tanaka, 2014; Tanaka et al., 2017). The systemic effectiveness of the electromyostimulation beyond the stimulation site is still unknown. In our previous study, RdPT, which is a new type of electromyostimulation, influenced the bone mechanical properties not only at the stimulated site but also at an unstimulated site (Tanaka et al., 2017). It is possible that multiple distant bones underwent neuronally regulated adaptation to the mechanical stimulation of a single bone (Rubin and Rubin, 2008; Sample et al., 2010); however, this topic requires further investigation (Sugiyama et al., 2010). In our current study, electromyostimulation did not show any effects on the mechanical properties of long bone diaphysis except in the stimulated femur (Table 1). This result suggests the limited remote effect of electromyostimulation on the distant long bones, which may be due to the delay or decay of neural signals over long distances from the stimulation site and/or due to a reduced response of cortical bones to the stimulation (Rubin et al., 2002).

The mechanical and/or microstructural properties of the vertebrae (L2 and L4) and femoral neck (Tanaka et al., 2017) were changed in response to electromyostimulation, thus suggesting that the remote effect of the stimulation could appear in trabecular bone but not in cortical bone. The richness of the nerve fiber in the proximal end of the femur has been reported previously (Mach et al., 2002), thus implying that the trabecular bone underwent neuronally regulated adaptation, which can also expand to the lumbar vertebrae by innervation (Buonocore et al., 2010). Connectivity exists via the network of nerves between each lumbar bone and femur or tibia (Rigaud et al., 2008). This 
suggests that electromyostimulation at the quadriceps could be selectively sensed by the nerves and could influence the metabolism, mechanical and/or microstructural properties of the distant bones via the neural network. It has been known that osteogenesis could be modulated by neurotransmitters from the sympathetic nervous system, which affects osteoblastic and osteoclastic activities (Takeda et al., 2002). The sensory nervous system can also influence bone formation by neuropeptides (Valentijn et al., 1997). Sympathetic nerves in the periosteum and the bone marrow cavity of the femur could be exposed by the electrical current of electromyostimulation (Takimoto and Tanaka, 2009). It is possible that neurotransmitters and neuropeptides are induced in the lumbar vertebrae by signals from the stimulated femur via the nervous system. This neurological signaling may contribute to the changes in mechanical properties and/or microstructural parameters in the L2 and L4 in the current study.

Although the general periodic stimulation patterns of electromyostimulation have been reported to increase bone mechanical properties (Lam and Qin, 2008; Qin et al., 2010; Tamaki et al., 2014), RdPT may have a higher capability of reinforcing bone mechanical properties. A geometric probability distribution was used for RdPT in this study. This probability is characterized by discreteness and memoryless, with which bones could avoid desensitization to the stimulation (Robling, et al., 2002), consequently providing a better response to the stimulation than PrPT (Tanaka et al., 2017). Bone mechanical properties depend on the protein-mineral ratio and enzymatic cross-linkage (Depalle et al., 2015; Paschalis et al., 2004; Saito et al., 2006; Viguet-Carrin et al., 2006). The increased stiffness of L2 by RdPT suggests that stimulation-induced neural signals promote the enzymatic cross-linking of collagen fibers at the lumbar vertebra despite the insignificant difference of BMD among the groups (Fig. 5a). Similarly, the strain energy should be affected by the cross-linking, although there was no significant difference among the groups (Fig. 3b), probably due to the limited displacement up to $4 \mathrm{~mm}$. On the other hand, the microarchitectual parameters of L2, i.e., BMC and TV, were significantly smaller in RdPT than those of control (Fig. 5c and e). The extent of collagen cross-linking of bone matrix affects the differentiation of bone cells (Ida et al., 2018). Therefore, we speculated that the cross-linking of collagen fibers could suppress bone remodeling and growth. Nevertheless, this hypothesis is applicable to L2, but not to L3 and L5, wherein the mechanical properties were unaffected (Table 2). Contrarily, L4 showed a decreased stiffness in RdPT stimulation. Unlike the other vertebrae connected to the femoral nerve or the sciatic nerve, only L4 is connected to both nerves (Rigaud et al., 2008), so that could be affected by different signal transduction pathway. Therefore, the RdPT effect is so site-dependent in the vertebrae. In any case, the positive effect of RdPT was limited to the stiffness of L2, and there were no obvious differences between RdPT and PrPT in the vertebrae.

It is noted that nonosteoporotic young rats were used in this study. These young rats are known to have adaptive bone remodeling; however, bone formation is more dominant than bone resorption. Although our data imply the similar effects of RdPT electromyostimulation in osteoporotic rat models, the data should be validated using them to confirm its applicability to the prevention of osteoporosis. In this study, we investigated the effect of stimulation on distant long bones at the diaphysis of the cortical bone. The epiphyseal trabecular bone of the long bones should be investigated for a remote effect from electromyostimulation. In addition to neural network effects, other possibly related factors (e.g., hormonal system, circulation system, immune system, and bone growth factor) may explain the results and should be investigated and discussed.

\section{Conclusion}

In conclusion, the remote effect of electromyostimulation on the mechanical and microstructural properties of bones in rats was investigated at a whole-body scale. Only RdPT at a femur increased the mechanical properties of the stimulated femoral diaphysis, but did not affect those of the diaphysis of the other long bones at unstimulated sites as well as PrPT. However, the lumbar vertebrae showed changes in mechanical and/or microstructural properties after each type of stimulation depending on the site of the vertebra. These findings suggest a possibility for the use of electromyostimulation as a novel physical therapy for osteoporosis in whole-body scale. However, the optimization of the stimulation conditions is still required for to give positive stimulation effects to all the bones toward clinical applications.

\section{Acknowledgments}

This research was partly supported by the Grant-in-Aid for Scientific Research (B) of the Ministry of Education, 
Science, Sports and Culture (Grant No. 15H03890). The author would also like to thank Kanazawa University (KU) Indonesian Ministry of Research, Technology, and Higher Education (RISTEKDIKTI) scholarship program.

\section{References}

Buonocore, M., Aloisi, A. M., Barbieri, M., Gatti, A. M. and Bonezzi, C., Vertebral body innervation: Implications for pain, Journal of Cellular Physiology, Vol.222, No.3 (2010), pp.488-491, DOI:10.1002/jcp.21996.

Caulkins, C., Ebramzadeh, E. and Winet, H., Skeletal muscle contractions uncoupled from gravitational loading directly increase cortical bone blood flow rates in vivo, Journal of Orthopaedic Research, Vol.27, No.5 (2008), pp.651-656, DOI:10.1002/jor.20780.

Depalle, B., Qin, Z., Shefelbine, S. J. and Buehler, M. J., Influence of cross-link structure, density and mechanical properties in the mesoscale deformation mechanisms of collagen fibrils, Journal of Mechanical Behavior Biomedical Material, Vol.52 (2015), pp.1-13, DOI:10.1016/j.jmbbm.2014.07.008.

Elkasrawy, M. N. and Hamrick, M. W., Myostatin (GDF-8) as a key factor linking muscle mass and bone structure, Journal of Musculoskeletal Neuronal Interact, Vol.10 (2010), pp.56-63.

Gourlay, M., Richy, F. and Reginster, J. -Y., Strategies for the prevention of hip fracture, The American Journal of Medicine, Vol.115 (2003), pp.309-317, DOI:10.1016/S0002-9343(03)00371-1.

Ida, T., Kaku, M., Kitami, M., Terajima, M., Rosales Rocabado J. M., Akiba, Y., Nagasawa, M., Yamauchi, M. and Uoshima, K., Extracellular matrix with defective collagen cross-linking affects the differentiation of bone cells, PLoS One. Vol.13, No.9 (2018), e0204306, DOI: 10.1371/journal.pone.0204306.

Lam, H. and Qin, Y. -X., The effects of frequency-dependent dynamic muscle stimulation on inhibition of trabecular bone loss in a disuse model, Bone, Vol.43 (2008), pp.1093-100, DOI:10.1016/j.bone.2008.07.253.

Mach, D. B., Rogers, S. D., Sabino, M. C., Luger, N. M., Schwei, M. J., Pomonis, J. D., Keyser, C. P., Clohisy, D. R., Adams, D. J., O'Leary, P. and Mantyh, P. W., Origins of skeletal pain: sensory and sympathetic innervation of the mouse femur, Neuroscience, Vol.113 (2002), pp.155-66, DOI:10.1016/S0306-4522(02)00165-3.

Matsunaga, T., Shimada, Y. and Sato, K., Muscle fatigue from intermittent stimulation with low and high frequency electrical pulses, Archives Physical Medicine and Rehabilitation, Vol.80, No.1 (1999), pp.48-53, DOI:10.1016/S0003-9993(99)90306-4.

Paschalis, E. P., Shane, E., Lyritis, G., Skarantavos, G., Mendelsohn, R. and Boskey, A. L., Bone fragility and collagen cross-links, Journal of Bone and Mineral Research, Vol.19, No.12 (2004), pp.2000-2004, DOI:10.1359/JBMR.040820.

Plochocki, J. H., Mechanically-induced osteogenesis in the cortical bone of pre- to peripubertal stage and peri- to postpubertal stage mice, Journal of Orthopaedic Surgery and Research, Vol.4, No.22 (2009), DOI:10.1186/1749-799X-4-22.

Qin, Y. X., Lam, H., Ferreri, S. and Rubin, C., Dynamic skeletal muscle stimulation and its potential in bone adaptation, Journal of Musculoskeletal Neuronal Interactions, Vol.10, No.1 (2010), pp.12-24.

Rigaud, M., Gemes, G., Barabas, M. -E., Chernoff, D. I., Abram, S. E., Stucky, C. L. and Hogan, Q. H., Species and strain differences in rodent sciatic nerve anatomy: implications for studies of neuropathic pain, Pain, Vol.136 (2008), pp.188-201, DOI:10.1016/j.pain.2008.01.016.

Robling, A. G., Hinant, F. M., Burr, D. B. and Turner, C. H., Improved bone structure and strength after long-term mechanical loading is greatest if loading is separated into short bouts, Journal of Bone and Mineral Research, Vol.17 (2002), pp.1545-1554, DOI:10.1359/jbmr.2002.17.8.1545.

Rubin, C., Turner, A. S., Mallinckrodt, C., Jerome, C., Mcleod, K. and Bain, S., Mechanical strain, induced noninvasively in the high-frequency domain, is anabolic to cancellous bone, but not cortical bone, Bone, Vol.30 (2002), pp.445-452, DOI:10.1016/S8756-3282(01)00689-5.

Rubin, J. and Rubin, C., Functional adaptation to loading of a single bone is neuronally regulated and involves multiple bones, Journal of Bone and Mineral Research, Vol.23, No.9 (2008), pp.1369-1371, DOI:10.1359/jbmr.09901.

Saito, M., Fujii, K., Mori, Y. and Marumo, K., Role of collagen enzymatic and glycation induced cross-links as a determinant of bone quality in spontaneously diabetic WBN/Kob rats, Osteoporosis International, Vol.17, No.10 (2006), pp.1514-1523, DOI:10.1007/s00198-006-0155-5.

Sample, S. J., Collins, R. J., Wilson, A. P., Racette, M. A., Behan, M., Markel, M. D., Kalscheur, V. L., Hao, Z. and 
Muir, P., Systemic effects of ulna loading in male rats during functional adaptation, Journal of Bone and Mineral Research, Vol.25, No.9 (2010), pp.2016-2028. DOI:10.1002/jbmr.101.

Shibata, Y., Ohsawa, I., Watanabe, T., Miura, T. and Sato, Y., Effects of physical training on bone mineral density and bone metabolism, Journal of Physiological Anthropology and Applied Human Science, Vol.22, No.4 (2003), pp.203-208, DOI:10.2114/jpa.22.203.

Sugiyama, T., Price, J. S. and Lanyon, L. E., Functional adaptation to mechanical loading in both cortical and cancellous bone is controlled locally and is confined to the loaded bones, Bone, Vol.46, No.2 (2010), pp.314-321, DOI:10.1016/j.bone.2009.08.054.

Takeda, S., Elefteriou, F., Levasseur, R., Liu, X., Zhao, L., Parker, K. L., Armstrong, D., Ducy, P. and Karsenty, G., Leptin regulates bone formation via the sympathetic nervous system, Cell, Vol.111, No.3 (2002), pp.305-317, DOI:10.1016/S0092-8674(02)01049-8.

Takimoto, T. and Tanaka, S. M., Noise electrical stimulation promotes alkaline phosphatase activity in regenerated bone, Japanese Journal Clinical Biomechanical, Vol.31 (2010), pp.181-186 (in Japanese).

Takimoto, T. and Tanaka, S. M., The use of electrical muscle stimulation to promote osteogenesis - analysis of current density distribution in bone by the finite element method, Journal Clinical Biomechanical, Vol.30 (2009), pp.21-26 (in Japanese).

Tamaki, H., Tomori, K., Yotani, K., Ogita, F., Sugawara, K., Kirimto, H., Onishi, H., Yamamoto, N. and Kasuga, N., Electrical stimulation of denervated rat skeletal muscle retards trabecular bone loss in early stages of disuse musculoskeletal atrophy, Journal of Musculoskeletal Neuronal Interact, Vol.14 (2014), pp.220-228.

Tamaki, H., Yotani, K., Ogita, F., Hayao, K., Nakagawa, K., Sugawara, K., Kirimoto, H., Onishi, H., Kasuga, N. and Yamamoto, N., Electrical stimulation of denervated rat skeletal muscle ameliorates bone fragility and muscle loss in early-stage disuse musculoskeletal atrophy, Calcified Tissue International, Vol.100 (2017), pp.420-430, DOI:10.1007/s00223-017-0250-y.

Tanaka, S. M., Effect of stimulation frequency on osteogenic capability of electrical muscle stimulation, Journal of Biomechanical Science and Engineering, Vol.9, No.3 (2014), pp.1-9, DOI:10.1299/jbse.14-00114.

Tanaka, S. M., Alam, I. M. and Turner, C. H., Stochastic resonance in osteogenic response to mechanical loading, FASEB Journal, Vol.17, No.2 (2002), pp.313-314, DOI: 10.1096/fj.02-0561fje.

Tanaka, S. M. and Kondo, K., Frequency and resting time dependencies of electrically-induced muscle contraction force, Journal of Biomechanical Science and Engineering, Vol.4, No.2 (2009), pp.201-211, DOI:10.1299/jbse.4.201.

Tanaka, S. M., Yorozuya, Y. and Takatsu, D., Random electromyostimulation promotes osteogenesis and the mechanical properties of rat bones, Annals Biomedical Engineering, Vol.45, No.12 (2017), pp.2837-2846, DOI:10.1007/s10439-017-1927-0.

Turner, C. H., Three rules for bone adaptation to mechanical stimuli, Bone, Vol.23, No.5 (1998), pp.399-407, DOI:10.1016/S8756-3282(98)00118-5.

Valentijn, K., Gutow, A. P., Troiano, N., Gundberg, C., Gilligan, J. P. and Vignery, A., Effects of calcitonin gene-related peptide on bone turnover in ovariectomized rats, Bone, Vol.21, No.3 (1997), pp.269-274, DOI:10.1016/S8756-3282(97)00142-7.

Viguet-Carrin, S., Garnero, P. and Delmas, P. D., The role of collagen in bone strength, Osteoporosis International, Vol.17, No.3 (2006), pp.319-36, DOI:10.1007/s00198-005-2035-9. 